\title{
Introduction: Ethics of Information Technology in Health Care
}

\begin{abstract}
:
Computer-based information and communication technologies continue to transform the delivery of health care and the conception and scientific understanding of the human body and the diseases that afflict it. While information technology has the potential to improve the quality and efficiency of patient care, it also raises important ethical and social issues. This IRIE theme issue seeks to provide a forum to identify, analyse and discuss the ethical and social issues raised by various applications of information and communication technology in medicine and health care. The contributions give a flavour of the extraordinarily broad landscape shaped by the intersection of medicine, computing and ethics. In fact, their diversity suggests that much more work is needed to clarify issues and approaches, and to provide practical tools for clinicians.
\end{abstract}

\section{Authors:}

Georg Marckmann, M.D., M.P.H.

University of Tübingen, Department of Medical Ethics, Schleichstr. 8, D-72076 Tübingen, Germany

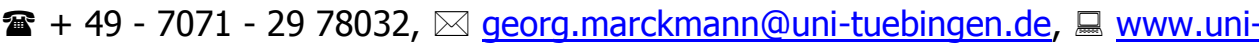

tuebingen.de/medizinethik

Relevant publications:

- Marckmann G. Recommendations for the Ethical Development and Use of Medical Decision Support Systems. Medscape General Medicine June 20, 2001. Available at: http://www.medscape.com/viewarticle/408143

- Marckmann G, Diagnose per Computer? Eine ethische Bewertung medizinischer Expertensysteme. Köln: Deutscher Ärzteverlag 2003

Kenneth W. Goodman, Ph.D.

Director, Bioethics Program, Co-Director, Ethics Programs, University of Miami, P.O. Box 016960 (M-825), Miami, FL 33101, USA

용 +1 305243 5723, $₫$ kgoodman@miami.edu, 매래 http://www.miami.edu/ethics/

Relevant publications:

Goodman KW (Hg.) Ethics, computing, and medicine. Informatics and the transformation of health care. Cambridge: Cambridge University Press 1998.

- $\quad$ Anderson JG, Goodman KW. Ethics and Information Technology. A Case-Based Approach to a Health Care System in Transition. New York: Springer 2002. 
Computer-based information and communication technologies continue to transform the delivery of health care and the conception and scientific understanding of the human body and the diseases that afflict it. In fact, modern medicine has become almost inconceivable without the use of computers. While information technology has the potential to improve the quality and efficiency of patient care, it also raises important ethical and social issues. Work on ethical issues at the intersection of medicine and computing has so far generated an ensemble of interesting and important questions:

What are appropriate uses of health information systems?

Who should use these systems?

What benefits and risks do these technologies have for patients?

How does information technology change the physician-patient relationship?

How does (and will) medical decision making change?

Perhaps most fundamentally: How does (and will) information technology transform the medical construction of the human body and disease?

Medical informatics has evolved as rapidly as any science in history, paralleling and relying on extraordinary advances in information collection, storage, analysis and transmission. Indeed, like sciences and technologies that precede it - biochemistry, microbiology, genetics and cell biology, pharmacology and so on - medical informatics is changing the standard of care. It is no longer fanciful to consider whether it might be blameworthy for a physician or an allied health professional to fail to use intelligent machines or their accoutrements in clinical practice and research.

Indeed and as ever, one should be forgiven for thinking that the science is progressing faster than the ethics. We are too soon able to do things before we know whether we ought to or not.

This is not a problem. It is rather a stage-setting in which we have the opportunity to use the tools of applied and theoretical ethics to track scientific change and, eventually, to guide it. If ethics lags too far behind, however, a science without moral underpinning risks losing touch with broadly shared human values. So we worry - we must worry when use of a new machine poses risks to patients; and we worry when failure to use the machine also may mean that a patient's care might be suboptimal, or that a patient might come to grief.

Ethical analysis of these concerns and tensions thus emerges as a moral imperative itself. Put differently, failure to scrutinize the expanding application of an evolving technology is itself blameworthy.

It follows that ongoing developments in the fields of information and (tele-)communication technology require continuous monitoring of ethical and social implications. This IRIE theme issue, "Ethics and Information Technology in Health Care," seeks to provide a forum to identify, analyse and discuss the ethical and social issues raised by various applications of information and communication technology in medicine and health care.

We have sought to construe the forum as broadly as possible, better to give a flavour of the extraordinarily broad landscape shaped by the intersection of medicine, computing and ethics. The contributions to this theme issue succeed nicely in making plain such breadth. In fact, their diversity suggests that much more work is needed to clarify issues and approaches, and to provide practical tools for clinicians. A patient-centered approach to medical care requires nothing less.

In the first contribution of this issue, Dirk Hagemeis ter investigates the effects of modern information technology (IT) on the working environment in hospitals and outpatient care and on the physician's decision-making process. On the one hand, IT certainly provides benefits for patient care by providing new computer-based diagnostic devices (e.g. the CAT scan), by improving the efficient storage, retrieval and exchange of patient data and as part of a basic infrastructure for integrated care networks. On the other hand, IT in medicine may also have negative consequences for patient care. In many cases, these effects are not a problem of IT per se but rather result from the complex interaction of IT with other structural determinants of a health care system, e.g., physician reimbursement systems. This makes clear that IT is primarily an instrument that can have both intended and unintended consequences, which in turn are heavily influenced by the specific policy framework. An electronic billing system, for example, might lead a physician to up- or downgrade the diagnostic code in order to increase his personal income. Some less visible, but nevertheless ethically important implications might occur if the electronically implemented diagnostic framework restricts the information input and the process of diagnostic reasoning. Users of IT 
in medicine therefore should be familiar with the specific strengths and limitations of the systems they are using.

Apart from storing and processing patient data, Information and computer technology in medicine can also be used to create virtual realities. Janne Lahtiranta and Kai Kimppa discuss in their contribution the use of anthropomorphized, human-like artefacts for teaching and training in medicine. Especially in intensive care and emergency medicine, students and residents learn clinical skills with manikins that show a realistic full-body anatomy. ${ }^{1}$ According to the Institute of Medicine (IOM) report "To err is human: Building a safer health system", between 44,000 and 98,000 people die in hospital each year as a result of medical errors that could have been prevented. ${ }^{2}$ As one measure to improve patient safety, the IOM suggests the use of simulation whenever possible:

"Health care organizations and teaching institutions should participate in the development and use of simulation for training novice practitioners, problem solving, and crisis management, especially when new and potentially hazardous procedures and equipment are introduced. ${ }^{13}$

As Lahtiranta and Kimppa point out, training with anthropomorphized artefacts may prevent harm and provides benefits for the patients. Consequently, we have an ethical obligation to promote training of clinical skills with manikins and dummies. However, these simulated realities will always fall short of the actualities of patient care. Therefore, medical students should be aware of these limitations and enjoy sufficient opportunity to interact with the complexity of real-world patients. Computer-based simulation can be a valuable supplement, but should never be a substitute of conventional bedside teaching.

Britta Schinzel is also concerned with the moral problems created by the use of constructive technologies. Modern imaging technologies like magnetic resonance imaging (MRI), positron emission tomography (PET) or functional MRI provide new and supposedly - realistic insights into the human body. However, these images are highly constructive artefacts that result from an extremely complicated

\footnotetext{
${ }^{1}$ Spence 1997

${ }^{2}$ Kohn et al. 2000

${ }^{3}$ Kohn et al. 2000, 179
}

and in many respects contingent combination of model driven algorithms, computations and visualizations. These epistemic observations become ethically relevant, if the images are used without reflection of their production process. Instead of depicting the world as it is (which in fact might be impossible), the images are heavily loaded with interpretations and create new meanings for "health," "disease," "normality," and "gender." Developing standardized atlases of the brain also elicits ethical issues given the plasticity and interand intra-individual variability of the cerebral structure and functioning. Due to their realistic appearance, these computed images create the illusory impression that certain differences between groups and populations are biologically fixed within the human brain. This can promote stereotypes and false dichotomies that are embedded in the seemingly "objective" results of scientific imaging techniques.

Information and communication technologies (ICT) in medicine are not only used to store, process, model, and transmit patient data. Innovative approaches try to integrate computer technology with the human body in order to obtain insights into the functioning of the body and to develop new diagnostic and therapeutic interventions. With these ICT implants, the use of computers in medicine seems to enter a new dimension, raising not only intriguing ethical issues but also anthropological issues. With the following two articles, we stay within the field of neuroscience research, but move from imaging to intervention.

Jens Clausen focuses on the ethical aspects of neuronal motor prostheses. Advances in the neurosciences and in micro system technology provide the potential to connect computer-systems with the human brain via brain-computer interfaces. This might offer new therapeutic perspectives especially for paralysed patients (e.g., hemiplegia). The goal is to bridge the interrupted nerve fibres with microtechnical devices and connect the cortex to an artificial limb or - even better - with the paralysed limb of the patient. On the one hand, braincomputer interfaces raise general ethical issues related to the protection of human subjects and the limits of a man-machine-integration. On the other, neuronal motor prostheses raise ethical issues that can be attributed to technological components themselves. Will the implanted electrodes of the input component that registers the cortical field potentials alter the patient's personality traits in an unacceptable way? Indeed, what should count as an "acceptable" alteration? Who bears responsibility for 
actions of the artificial limb that result from an indissoluble interaction between the patient's brain and the decoding algorithm? Will the (probably) wireless output component allow unwanted external control or interference? Risks for patients due to malfunctioning of the system must be balanced against the benefits of restored limb function. It certainly will require many year of development and clinical testing before neuronal motor prostheses will become available for patients. Nevertheless, ethical analysis should accompany these technological developments right from the start as part of the timely anticipation of possible adverse effects on the patients.

Elisabeth Hildt discusses another application of ICTimplants in the neurosciences: With electrodes implanted in the brain, deep brain stimulation provides the possibility to influence the functioning of certain brain regions, e.g. for tremor control in patients with Parkinson's disease. Other potential applications of neurostimulation include the treatment of severe neuropsychiatric disorders like obsessive-compulsive disorders. Like many other invasive medical technologies, deep brain stimulations involve benefits (e.g. improvement of tremor) and risks (e.g. infections, intracranial haemorrhage, adverse neuropsychological effects). However, the effects of deep brain stimulation deserve special attention because they interact with brain structures that might influence the patient's personality and character traits: How far will the patient be able to control the effects of neurostimulation? Will these changes be reversible? This calls for a thorough assessment of clinical benefits and adverse effects, especially in the long term. With regard to other possible applications - e.g. enhancement of cognitive functions - Hildt concludes that deep brain stimulation should be restricted to severe disorders with a well-known pathophysiological basis for which there are no other less invasive treatments with comparable effectiveness.

Michael Nagenborg and Mahha El-Faddagh conclude this issue with a paper on the availability, appropriate use and ownership of genetic information. In this case, ethical issues arise from the application of computer technology for processing a specific type of medical information. While genetic information plays an important role in the diagnosis of hereditary diseases, it also provides opportunities to select effective therapeutic interventions according to the specific genotype of the patient. However, other players like employers or insurance companies are interested in genetic information. This gives rise to the question: Who has legitimate access to the genetic information? Who owns or controls the genetic information? Nagenborg and El-Faddagh apply the concept of different information spheres, and argue that the usage of genetic information should be confined to the medical sphere. Based on a Kantian concept of ownership the individual is the "owner" of information about his genes in the sense that he has the right to exclude other people form using this very personal information. Hence, information infrastructures in health care should be organized in a way that preserves the boundaries of the medical sphere and precludes unauthorized usage of genetic information by non-medical institutions. As the authors point out, this will be a difficult task given increasing economic pressures on the world's health care systems.

The contributions in this IRIE issue present a colourful picture of the rapidly expanding field of information and communication technology in medicine and health care. New ethical issues arise depending on the specific context in which computer technology is applied. The articles here provide several examples to illustrate the point that it is not ICT per se that raises ethical issues, but rather the connection of ICT to certain concepts, types of medical information and other technologies. Especially in the field of neuroscience research, the increasing integration of human and machine with ICT-implants will pose intriguing epistemological, ethical and anthropological questions. Depending on the specific context, ethical analysis of ICT applications in medicine therefore will increasingly have to combine insights and approaches from several different disciplines.

\section{References}

Kohn LT, Corrigan JM, Donaldson MS (eds.). To err is human. Building a safer health system. Washington: National Academy Press; 2000.

Spence $A A$. The expanding role of simulators in risk management. $\mathrm{Br} J$ Anaesth 1997;78(6):633-4. 